\section{A) Check for updates}

Cite this: Food Funct., 2022, 13, 1725

\title{
Long-term exclusive enteral nutrition remodels the gut microbiota and alleviates TNBS-induced colitis in mice $\uparrow$
}

\author{
Gengfeng Li, (iD Xiaohan Wu, Xiang Gao, Ritian Lin, Liang Chen, Mingming Sun, \\ Junwan Jia, Zhanju Liu, Leilei Fang* and Wei Wu (D) *
}

\begin{abstract}
Background: Exclusive enteral nutrition (EEN) provides an effective strategy for the induction of clinical remission in pediatric Crohn's disease. However, the feasibility of long-term EEN in the management of disease and the underlying mechanism whereby long-term EEN prevents intestinal inflammation are still not fully understood. Methods: Paired male and female adult wild-type (WT) mice were mated to breed littermates, and these pups were then weaned at 3 weeks of age and randomly allocated into regular diet (RT) feeding group and EEN feeding group (Peptisorb; NUTRICIA), respectively. After feeding until adulthood at the age of 8 weeks, mice were sacrificed and phenotypic analysis of immune cells in spleens and mesenteric lymph nodes (MLNs) was performed by flow cytometry. Fecal pellets were also collected to determine the levels of immunoglobulins and gut microbiota by ELISA and 16S rRNA sequencing. The role of long-term EEN in the development of colitis and its underlying mechanisms were evaluated in a TNBS-induced colitis model in mice. Results: Feeding with EEN decreased the percentages of IgA- and IgG-coated bacteria and the levels of soluble IgA and IgG in the feces of EEN-feeding mice compared with the controls, but did not affect the compositions of different immune cells including $\mathrm{CD} 4^{+}, \mathrm{CD} 8^{+}$ $\mathrm{T}$ cells and $\mathrm{B} 22 \mathrm{O}^{+} \mathrm{B}$ cells in the spleens and MLNs. An in-depth analysis of the gut microbiota revealed a decrease of the general diversity of the gut microbiota, but a significant change of the composition of the gut microbiota after EEN feeding, characterized by an increase of the beneficial bacteria including Bacteroides, Parabacteroides, and Alistipes, but a decrease of the detrimental bacteria such as Escherichia-Shigella. Moreover, we found that EEN feeding markedly improved intestinal inflammation in the TNBS-induced colitis model compared to RT feeding, as evidenced by decreased levels of inflammatory cytokines and fecal soluble immunoglobulins and improved microbial community composition. Conclusions: Our data indicate that long-term EEN feeding remodels the composition of gut microbiota and alleviates intestinal mucosal inflammation. It provides new guidance using EEN for the management of gut inflammation.
\end{abstract}

Received 24th October 2021, Accepted 17th December 2021 DOI: $10.1039 / \mathrm{d} 1 \mathrm{fo} 03579 \mathrm{~g}$ rsc.li/food-function

\section{Introduction}

Crohn's disease (CD), one subset of inflammatory bowel diseases (IBD), is a chronic relapsing intestinal disease characterized by recurrent diarrhea, abdominal pain and weight loss. In spite of great advances in the studies of pathogenesis and therapy, CD still represents a big challenge owing to its considerable morbidity associated with the progressive nature of the disease and huge heterogeneity leading to treatment

Center for IBD Research, Department of Gastroenterology, Shanghai Tenth People's Hospital, School of Medicine, Tongji University, No. 301 Yanchang Road,

Shanghai 200072, China. E-mail: wuwei_1125@126.com, leileilily728@sina.com $\dagger$ Electronic supplementary information (ESI) available. See DOI: 10.1039/ d1fo03579g failure. It is generally accepted that a confluence of different factors including genetics, aberrant immune responses, microbiota and environmental factors leads to the pathogenesis of CD. ${ }^{1}$ A definitive change of gut microbiota ${ }^{2,3}$ in conjunction with a dysfunctional crosstalk between the host and the gut microbiome is considered as a defining event in the development of CD. Dietary factors, as the most associated environmental factors, play an essential role in the gut microenvironment by regulation of the gut microbiota composition, ${ }^{4}$ intestinal epithelial barrier function ${ }^{5}$ and host immunity, participating in the maintenance of intestinal homeostasis.

Therefore, dietary manipulation may represent a promising treatment modality for patients with $\mathrm{CD}$ relative to existing immune-based therapies. Different dietary strategies including exclusive enteral nutrition (EEN), partial enteral nutrition 
(PEN), Crohn's disease exclusion diet (CDED), specific carbohydrate diet (SCD), low fermentable oligosaccharides, disaccharides, monosaccharides and polyols (FODMAP) diet have been attempted and showed promise for CD patients under different conditions, with EEN showing the strongest evidence for the induction of remission in pediatric CD. ${ }^{6} \mathrm{EEN}$, as its name implies, involves exclusive use of a complete liquid diet with deprivation of any other foods usually for $6-8$ weeks, no more than 12 weeks. EEN is a specialized liquid nutritional formula which can be classified as polymeric, semi-elemental or elemental formulas depending on whether the protein is delivered as intact proteins or individual amino acids. Owing to its evident therapeutic effects, avoidance of side effects of corticosteroids and improved individual growth, EEN is recommended as the first-line therapy in the management of pediatric $\mathrm{CD}^{7}$ In addition to the induction of remission for pediatric $\mathrm{CD}$, EEN has also been demonstrated to prevent disease progression to complications and as supportive therapy in those with malnutrition or in perioperative settings.

One crucial mechanism of action of EEN lies on the modulation of gut microbiota. ${ }^{8}$ Extensive and sustained changes in the gut microbiota composition and functionality have been observed in children with CD during EEN. ${ }^{9-11}$ Paradoxically, EEN even decreases the microbial diversity, which is a prominent characteristic of the dysbiotic microbiome in IBD. ${ }^{9,10,12}$ The mechanism may involve the depletion of existing gut bacteria and restructuring of the new gut microbiome. ${ }^{13}$ Apart from regulation of the gut microbiome, other mechanisms of EEN on CD have been elucidated, including the modulation of systemic and local immune responses and maintenance of the intestinal barrier integrity. Feeding with EEN significantly downregulates the levels of mucosal pro-inflammatory cytokines and corrects the pro- and anti-inflammatory imbalance to promote mucosal healing. ${ }^{14-16}$ Direct evidence comes from an in vitro study, demonstrating that the polymeric formula inhibits an epithelial IL-8 response through the modulation of the NF-kB signaling pathway. ${ }^{17}$ Other in vitro studies also showed that polymeric formula can directly reverse the increased epithelial permeability on TNF- $\alpha$ insults. ${ }^{18-20}$ Moreover, components added in the enteral nutrition such as glutamine, arginine, and vitamin D3 possessing anti-inflammatory capacities may also confer a role in the induction of remission. ${ }^{21}$

EEN requires firm compliance to avoid all other foods and there is a high rate of relapse once habitual diets are reintroduced after the induction of remission by EEN ${ }^{22}$ suggesting the major mechanism leading to response is attributed to the principle of exclusivity. ${ }^{23,24}$ Childhood-onset CD patients benefit a lot from EEN treatments as a result of weakened aggressive disease course and improved growth and development. Despite the evident therapeutic effects EEN exerts in induction of remission, the bad palatability and unsustainability sometimes discourages continuous application. However, there is no doubt that long-term adherence to EEN may maximally achieve benefits in $\mathrm{CD}$. Therefore, we reasoned if longterm EEN is feasible and meets the caloric requirements for development if fed throughout the growth stage. Besides, we aimed to find direct evidence indicating whether restructuring of gut microbiota by long-term EEN could improve the tolerance against TNBS-induced colitis in adulthood.

In the current study, we investigated whether long-term EEN substitution for regular diet through weaning to adulthood could meet the needs for growth and development and affect the development of the immune system and composition of the gut microbiota. We found that feeding with EEN significantly decreased fecal IgA- and IgG-coated bacteria and the levels of soluble IgA and IgG as well. Despite the general diversity of the gut microbiota being decreased after EEN treatment, the composition of the gut microbiota significantly changed, characterized by an increase of some beneficial bacteria including Bacteroides, Parabacteroides, and Alistipes in conjunction with reduction of detrimental bacteria such as Escherichia-Shigella. Furthermore, a TNBS-induced colitis model was established and EEN-treated mice developed less severe colitis upon TNBS insults relative to regular diet (RT) feeding mice. The levels of IgA and IgG were decreased and beneficial bacteria were increased in the feces of EEN-treated colitic mice. Collectively, our data demonstrate that long-term EEN does not impact the development of the immune system and meets the needs for growth and development. Regulation of the microbial composition by long-term EEN confers protective effects against colitis.

\section{Materials and methods}

\section{Animals and the experimental design}

Mice (C57BL/6J) were purchased from Shanghai SLAC Laboratory Animal Co., Ltd (Shanghai, China), and bred and reared under specific pathogen-free facilities at the Experimental Animal Center of Tongji University (Shanghai, China). All mice had free access to filtered air, sterile water and autoclaved food in individually ventilated cages with a 12-hour light-dark cycle. All animal experiments in this study were approved by the Institutional Animal Care and Use Committee of Tongji University.

After acclimatization for a minimum period of 7 days, several pairs of male and female WT mice were mated at the same time to acquire littermates (about 20-30 mice). All pups were weaned at the age of 3 weeks, separated according to the sex and randomly allocated into RT or EEN feeding groups. Peptisorb enteral nutritional suspensions (NUTRICIA, Wuxi, China) were provided as a sole nutrient/water source with the exclusion of standard mouse chow in the EEN group, whereas water and standard mouse chow were supplied ad libitum in the RT group. The standard mouse chow diet was purchased from Jiangsu Xietong Pharmaceutical Bioengineering Co., Ltd (cat\# 1010084; Nanjing, China). Detailed ingredients of the EEN diet and standard mouse chow diet are shown in ESI Tables 1 and $2, \uparrow$ respectively. Two groups of mice were fed with RT or EEN until adulthood at the age of 8 weeks. These adulthood mice were then used to establish an experimental 
colitis model through a rectal administration of TNBS as described previously. ${ }^{25}$

\section{Flow cytometry analysis of the immune cell composition in the spleens and mesenteric lymph nodes (MLNs)}

Spleens and MLNs were collected and ground thoroughly into homogenates by using frosted glass slides. After hypotonic lysis to remove the red blood cells, the tissue homogenates were centrifuged to discard the supernatant. The pellets were resuspended and filtered through a $70 \mu \mathrm{m}$ filter to acquire single-cell suspensions. The total cells were then collected and incubated with fluorescein-conjugated antibodies against mouse CD4, CD8, B220 and CD11c and a live/dead dye (L10119, Invitrogen) for $30 \mathrm{~min}$ at $4{ }^{\circ} \mathrm{C}$. Flow cytometry was performed using a fluorescence-activated cell sorting (FACS) Canto $^{\text {TM }}$ II (BD Biosciences; San Diego, CA, USA) according to the manufacturer's instructions. Data analysis was carried out by using FlowJo VX software (Tree Star, Inc.; Ashland, OR, USA).

\section{Measurement of fecal IgA- and IgG-coated bacteria by flow cytometry}

IgA- and IgG-coated bacteria in the feces were analyzed as described previously. ${ }^{26}$ Briefly, fecal pellets were collected from both RT- and EEN-feeding mice at 10 am and homogenized by bead beating. After centrifugation to separate large particles, the supernatants containing fecal bacteria were collected and washed with $1 \mathrm{ml}$ PBS containing 1\% bovine serum albumin. Bacterial pellets were then incubated with blocking buffer. Fecal homogenates were then stained with PE-conjugated anti-mouse IgA, FITC-conjugated anti-mouse IgG and a live/dead dye for $30 \mathrm{~min}$ on ice. Flow cytometry analysis of fecal IgA- and IgG-coated bacteria was performed using a FACS Canto $^{\text {TM }}$ II (BD Biosciences; San Diego, CA, USA) according to the manufacturer's instructions. All data were analyzed using FlowJo VX software (Tree Star, Inc.; Ashland, OR, USA).

\section{Extraction and polymerase chain reaction (PCR) amplification of fecal 16S rRNA}

16S rRNA from mouse fecal samples was extracted using an E. Z.N.A. ${ }^{\circledR}$ Soil DNA Kit (Omega Bio-Tek, Norcross, GA, USA) according to the manufacturer's instructions. Fecal DNA quality and quantity were determined using a NanoDrop 2000 instrument (Thermo Fisher Scientific, Massachusetts, USA). The V4-V5 variable regions of the bacterial 16S rRNA gene were amplified by PCR with universal primer pairs, 515F 5'barcode-GTGCCAGCMGCCGCGG-3' and 907R ${ }^{\prime}$ 'CCGTCAATTCMTTTRAGTTT-3', where barcode is an eight-base sequence unique to each sample. PCR reactions were performed in triplicate using a $20 \mu \mathrm{L}$ mixture containing $4 \mu \mathrm{L}$ of 5 $\times$ FastPfu buffer, $2 \mu \mathrm{L}$ of $2.5 \mathrm{mM}$ dNTPs, $0.8 \mu \mathrm{L}$ of each primer $(5 \mu \mathrm{M}), 0.4 \mu \mathrm{L}$ of FastPfu polymerase, and $10 \mathrm{ng}$ of template DNA. The PCR reaction programs were as follows: $95{ }^{\circ} \mathrm{C}$ for $2 \mathrm{~min}$, followed by 25 cycles at $95^{\circ} \mathrm{C}$ for $30 \mathrm{~s}, 55^{\circ} \mathrm{C}$ for $30 \mathrm{~s}$, and $72{ }^{\circ} \mathrm{C}$ for $30 \mathrm{~s}$ and a final extension at $72{ }^{\circ} \mathrm{C}$ for $5 \mathrm{~min}$. Amplicons were extracted from $2 \%$ agarose gels and purified using the AxyPrep DNA Gel Extraction Kit (Axygen Biosciences, Union City, CA, USA) according to the manufacturer's instructions and quantified using QuantiFluor ${ }^{\mathrm{TM}}-\mathrm{ST}$ (Promega, USA).

\section{Library construction and 16S rRNA sequencing}

Purified PCR products were quantified using Qubit® 3.0 (Life Invitrogen) and every twenty-four amplicons whose barcodes were different were mixed equally. The pooled DNA product was used to construct an Illumina Pair-End library following Illumina's genomic DNA library preparation procedure. The amplicon library was then paired-end sequenced $(2 \times 250)$ on an Illumina MiSeq platform (Shanghai Biozeron Co., Ltd) according to the standard protocols.

The QIIME software package (version 1.8.0) was used to analyze the raw Illumina MiSeq sequencing data. ${ }^{27}$ The reads with low quality were discarded if the quality scores were below 20 or sequence lengths below $400 \mathrm{bp}$. The unique sequences among these remaining reads were used to define the operational taxonomic units (OTUs) using Usearch (version 7.1, http://drive5.com/uparse/) with a threshold of $97 \%$ similarity. The taxonomic identities of the phylotypes were determined using the Ribosomal Database Project (RDP) Classifier (version 2.2, http://sourceforge.net/projects/rdpclassifier/) at a confidence threshold of $70 \%$. Alpha diversity analysis and beta diversity analysis were obtained using QIIME. Dissimilarities in the community composition were shown by principal coordinates analysis (PCoA) based on the weighted UniFrac distance metric. Furthermore, Linear Discriminant Analysis Effect Size analysis (LEfSe) was used to detect the dominant bacterial community differences among groups.

\section{Establishment of an acute TNBS-induced colitis model in mice}

A TNBS-induced colitis model in mice was established as described previously. ${ }^{25}$ In short, adulthood mice from both RT and EEN feeding groups were fasted for $24 \mathrm{~h}$ but with access to drinking water ad libitum in order to eliminate as much feces as possible. TNBS was prepared by dissolving 5\% (w/v) TNBS (P2297, Sigma-Aldrich) with an equal volume of absolute ethanol to acquire a working solution of $2.5 \%(\mathrm{w} / \mathrm{v})$ TNBS in $50 \%$ ethanol. After anesthetizing with $1.25 \%$ pentobarbital sodium intraperitoneally, mice were administered $150 \mu \mathrm{L}$ of $2.5 \%(\mathrm{w} / \mathrm{v})$ TNBS solution (dissolved in 50\% ethanol) intrarectally using a $3.5 \mathrm{~F}$ polyethylene catheter. Another two groups were treated with $50 \%$ ethanol intrarectally as the controls.

Over the period of the study, characteristics of acute TNBSinduced colitis including body weight, diarrhea, and bloody stools were recorded daily. All mice were sacrificed on day 6 and the distal colon tissues were removed, fixed in $4 \%$ paraformaldehyde, embedded in paraffin, sectioned, and stained with hematoxylin and eosin.

\section{Hematoxylin and eosin (H\&E) staining}

Samples of the colon tissues were collected, fixed with $4 \%$ paraformaldehyde and embedded in a paraffin block followed by cutting into $5 \mu \mathrm{m}$ slices. These sections were then transferred onto glass slides for next use. After deparaffinization and rehy- 
dration, the tissues were stained with Mayer's hematoxylin for 5 min and then rinsed in tap water to remove the excess dyes, followed by differentiation with a weak acid solution and bluing in mildly alkaline water. Then the tissues were counterstained with eosin. Finally, the slides were dehydrated, cleared with xylene and mounted in synthetic resin. Photographs of all specimens were observed under an optical microscope (AF6000, Leica; Wetzlar, Germany). Criteria for the histopathological scoring of the colon sections are listed in ESI Table 3.†

\section{Statistical analysis}

Data were expressed as mean \pm SEM, and analyzed using GraphPad Prism 7 software (GraphPad Software; CA, USA). Statistical comparisons were performed using unpaired Student's $t$-test, paired Student's $t$-test and one-way analysis of variance (ANOVA). Statistical significance was defined as follows: ${ }^{*} P<0.05,{ }^{*} P<0.01$ and ${ }^{* * *} P<0.0001$.

\section{Results}

\section{EEN feeding does not affect the immune cell development}

In order to investigate whether long-term EEN feeding would affect immune cell development under physiological conditions, we fed 3-week-old pups with RT or EEN from weaning to adulthood (Fig. 1A). Both groups of 8-week-old mice have similar appearance and normal individual growth and development. These mice were then sacrificed and the colons, spleens and MLNs were collected for the next analysis. Spleens and MLNs were ground into homogenates and single-cell suspensions were prepared for flow cytometric analysis. There were no differences in the percentages of $\mathrm{CD}^{+}, \mathrm{CD}^{+}{ }^{+}$cells, B220 ${ }^{+}$ $B$ cells and CD11c ${ }^{+}$DCs in spleens and MLNs between the two groups (Fig. 1B and C). Furthermore, the colon tissues were obtained and total RNA was extracted to detect inflammatory cytokine expression. We found that there was a decreasing trend in the expressions of IFN- $\gamma$, IL-17A, IL-10 and Foxp3 mRNA in the colon tissues of EEN feeding mice but no difference from that in the RT feeding mice (data not shown). These data suggest that EEN feeding meets the caloric needs and does not affect immune cell development during the growth of mice.

\section{EEN feeding decreases the fecal levels of IgA- and IgG-coated bacteria and soluble immunoglobulins}

As IgA- and IgG-coated bacteria have been implicated in the regulation of gut homeostasis, ${ }^{28,29}$ we then assessed IgA- and IgG-coated gut bacteria in the feces from both RT and EEN feeding mice. Impressively, $\mathrm{IgA}^{+}$and $\mathrm{IgG}^{+}$bacteria were significantly decreased in the EEN feeding group compared with those in the RT feeding group (Fig. 2A and B). Moreover, we determined the levels of soluble immunoglobulins by ELISA. As expected, the levels of soluble IgA and IgG decreased in the feces of EEN feeding mice compared with that of the controls (Fig. 2C). Taken together, these results indicate that EEN feeding reduces the fecal levels of immunoglobulin-coated bacteria and soluble immunoglobulins.

\section{EEN feeding alters the diversity and structure of the gut microbiota}

The above results show that EEN feeding decreased the fecal levels of IgA- and IgG-coated bacteria, leading to the hypothesis that EEN feeding may alter the gut microbiota composition. Therefore, we sought to investigate whether the diversities and compositions of gut microbiota were altered concomitantly with EEN feeding. To this end, we collected the colonic fecal samples before and after RT or EEN feeding from the two groups. Bacterial 16S rRNA sequencing was performed to characterize the effects of EEN on the changes of the gut microbiota. According to the Shannon-Wiener curve and rankabundance distribution curve shown in Fig. 3A and B, it was suggested that the sequence depth was sufficient for capturing the majority of OTUs and that the amount of sequencing data was enough to reflect microbial information in all samples. Microbial within-community (alpha, $\alpha$ ) diversity analysis, which was reflected by the Chao and Shannon indexes, revealed increased richness and diversity after RT feeding at the age of 8 weeks. However, the EEN feeding mice showed decreased Chao and Shannon indexes relative to the RT feeding group, suggesting a reduced $\alpha$-diversity after EEN feeding, consistent with previous studies as described above (Fig. 3C and D).

In addition, UniFrac distance-based principal coordination analysis (PCoA) and nonmetric multidimensional scaling (NMDS) were adopted to evaluate the variance of between-community (beta, $\beta$ ) diversity among different groups. It was evident that the OTUs from the B-RT group overlapped with those from the B-EEN group based on two-dimensional PCoA and NMDS plots. After feeding with RT or EEN, the OTUs from the A-RT group were clustered distinctly from that of the A-EEN group, suggesting significant separation in the gut microbiota structure between the two groups (Fig. 3E and F). Collectively, these data indicate that EEN feeding rigorously changes fecal microbial $\alpha$ and $\beta$-diversity compared with RT feeding.

\section{Pivotal phylotypes of the gut microbiota responding to EEN feeding}

To illustrate the differences in the microbiota composition among different groups in detail, we further analyzed the gut bacterial compositions at the phylum and genus levels. At the phylum level, 98-99\% of the gut microbial community was dominated by Bacteroidetes, Firmicutes, Proteobacteria and Actinobacteria in these four groups. Of note, we observed that the EEN feeding group displayed increased levels of Proteobacteria, but decreased levels of Actinobacteria relative to the RT feeding group, though the two strains occupied only small proportions. In addition, there was no difference in the proportion of Bacteroidetes and Firmicutes among the four groups (data not shown). However, in terms of lower taxonomic levels, EEN feeding did alter the community structure as shown by the microbial community bar plot and heatmap 
A

Regular Diet

Exclusive Enteral Nutrition

\section{Breastfeeding}

Breastfeeding $\downarrow$
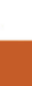

Standard mouse chow and water

Exclusive Enteral Nutrition

\begin{tabular}{ccc}
$\stackrel{\text { Birth }}{\perp}$ & Weaning & $\underset{\perp}{\perp}$ \\
\hline 0 & 3 weeks & Adulthood \\
\hline
\end{tabular}

B
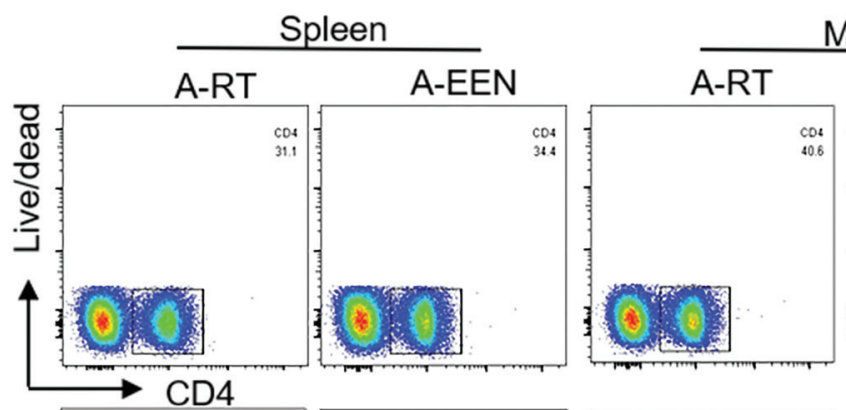

MLN
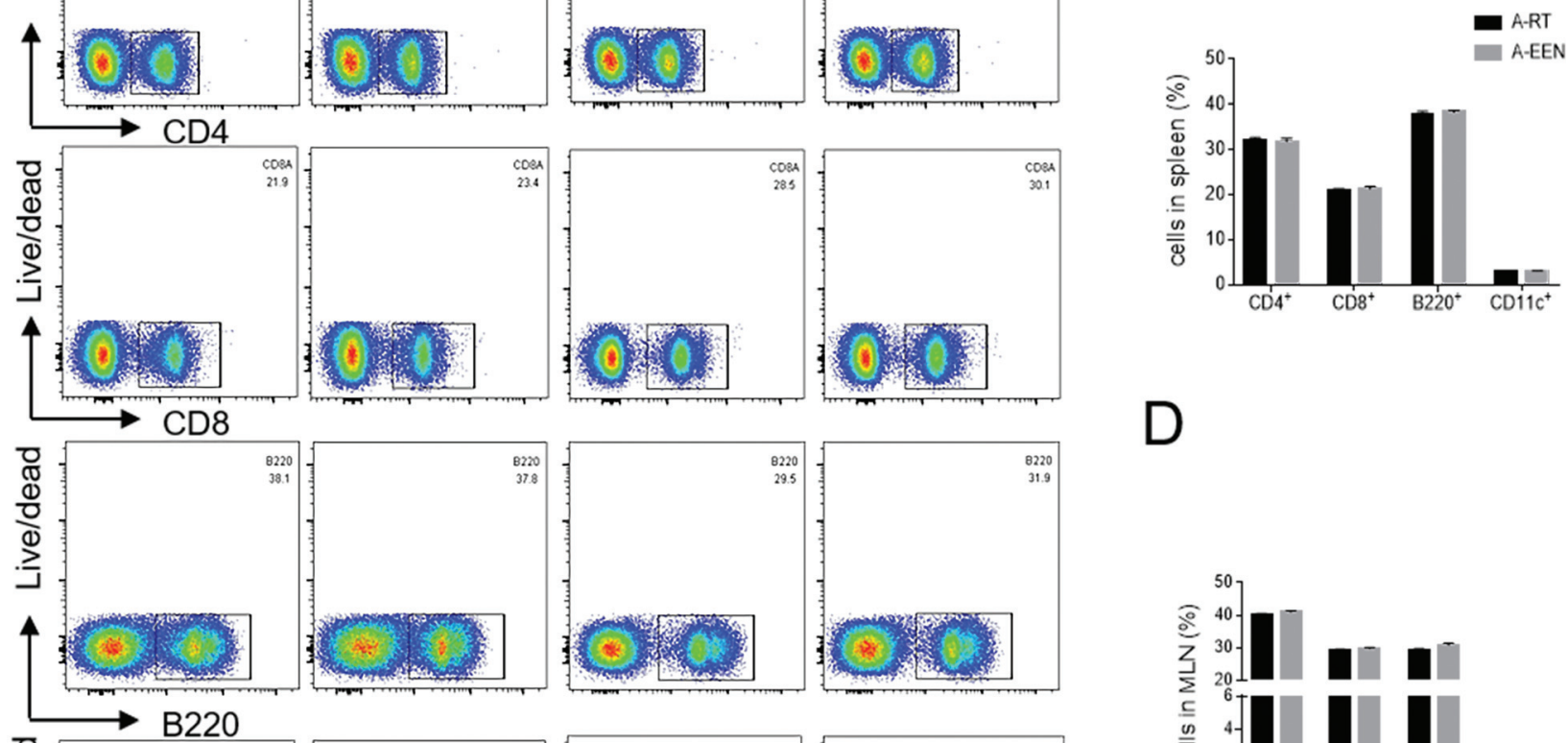

D
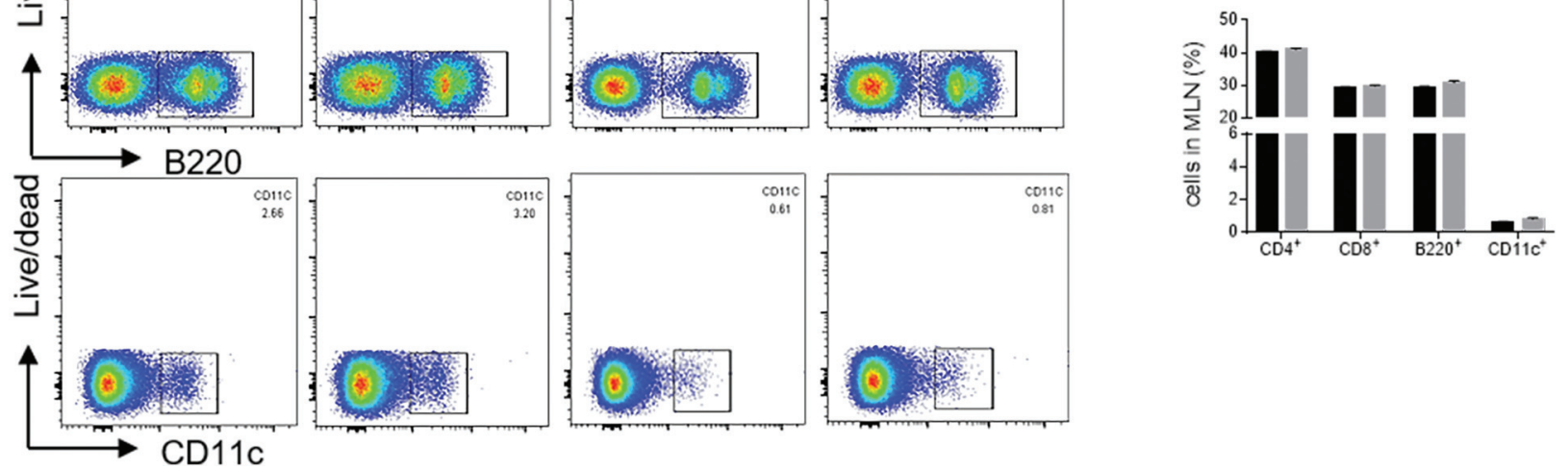

Fig. 1 EEN feeding does not affect immune cell development. Neonatal mice received breastfeeding until 3 weeks of age, and were then weaned and randomly allocated into the RT and EEN feeding groups, respectively, for continuous feeding until adulthood. (A) Schematic diagram of an experimental design. At the age of 8 weeks, both RT $(n=4)$ and $\operatorname{EEN}(n=6)$ feeding groups of mice were sacrificed and the spleens and MLNs were collected and ground into homogenates to acquire single-cell suspensions. (B) Flow cytometry analysis of $\mathrm{CD} 4^{+}, \mathrm{CD} 8^{+} \mathrm{T}^{\mathrm{c}}$ cells, $\mathrm{B} 22 \mathrm{O}^{+} \mathrm{B}$ cells and $\mathrm{CD} 11 \mathrm{C}^{+}$dendritic cells in the spleens and MLNs. (C and D) Percentages of $\mathrm{CD} 4^{+}, \mathrm{CD} 8^{+} \mathrm{T}$ cells, B220 $\mathrm{O}^{+} \mathrm{B}$ cells and $\mathrm{CD} 11 \mathrm{C}^{+}$dendritic cells in the spleens and MLNs. Data are representative of 3 independent experiments. A-RT, after RT; A-EEN, after EEN.

at the genus level in Fig. 4A and B. Previous studies have demonstrated an even more dysbiotic state in the gut microbiota of pediatric CD patients, concomitant with disease remission after EEN treatment. According to our present results, the gut microbiota composition vigorously changed within the phylum Bacteroidetes (Fig. 4B).
Since dysbiosis is a marked signature of IBD, we then questioned whether IBD-associated bacteria could also be modulated by EEN feeding. For example, the genera Alistipes, Odoribacter and Rikenella were reported to be decreased in IBD patients and administration with Alistipes or Parabacteroides ameliorates DSS-induced colitis in mice. ${ }^{30-32}$ Moreover, the 
A

B
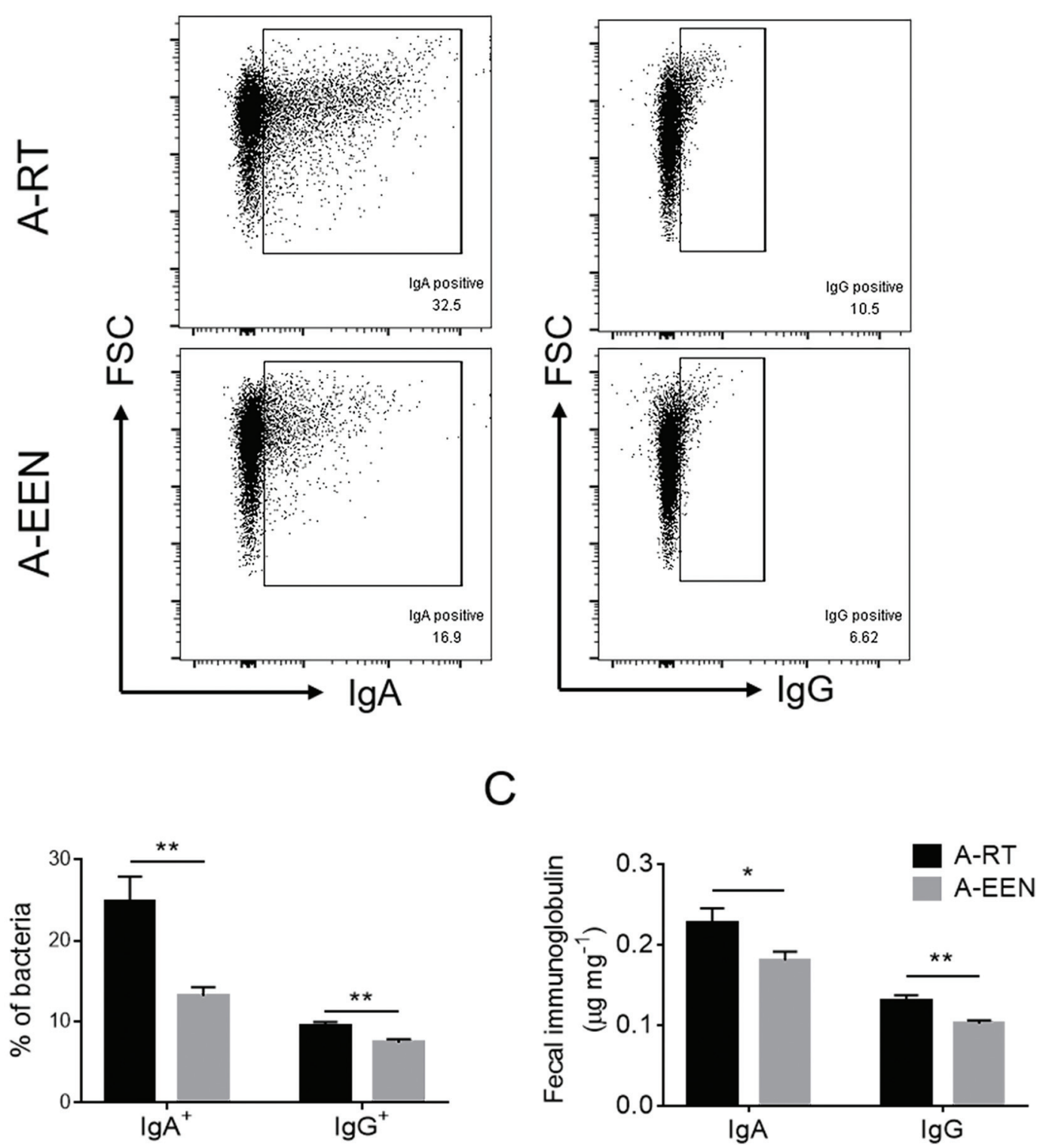

Fig. 2 EEN feeding decreases the fecal levels of IgA- and IgG-coated bacteria and soluble immunoglobulins. Fecal samples were harvested from both RT and EEN feeding mice, and fecal IgA- and IgG-coated bacteria were determined by flow cytometry. Fecal levels of soluble IgA and IgG were measured by ELISA. (A) FACS profiles of IgA- and IgG-coated bacteria in the feces. (B) Bar chart of IgA- and IgG-coated bacteria in the feces. (C) The levels of soluble IgA and IgG in the feces. ${ }^{*} P<0.05$ and ${ }^{*} P<0.01$. Data are representative of 3 independent experiments.

genus Allobaculum has the capacity to catabolize tryptophan into indole derivatives, which further participate in modulating intestinal immune responses. ${ }^{33}$ The genera Odoribacter and Coriobacteriaceae UCG-002 are found to be suppliers of SCFAs and decreased abundance is associated with host inflammation. ${ }^{34,35}$ We thus compared the relative abundance of several specific bacteria at the genus level among different groups. Intriguingly, we observed that the levels of beneficial bacteria including Bacteroides, Parabacteroides, Alistipes, Allobaculum, Odoribacter, Coriobacteriaceae UCG-002 and Rikenella increased in various degrees (Fig. 5A-G), while the abundances of opportunistic pathogens such as
Mucispirillum and Escherichia-Shigella, which are correlated with intestinal inflammatory diseases, were markedly decreased (Fig. 5H and I).

To identify the differentially abundant taxa with biological consistency associated with EEN feeding, we performed the linear discriminant analysis effect size (LEfSe) analysis to examine the differential microbial flora from phylum to genus among four groups. As shown in Fig. 6A and B, Rikenellaceae, Alistipes, Allobaculum, Odoribacter and Rikenella represented the most important components in the EEN feeding group of mice, which were significantly different from those in the RT feeding mice. In summary, these results obtained in the 
A

Shannon-Wiener curve

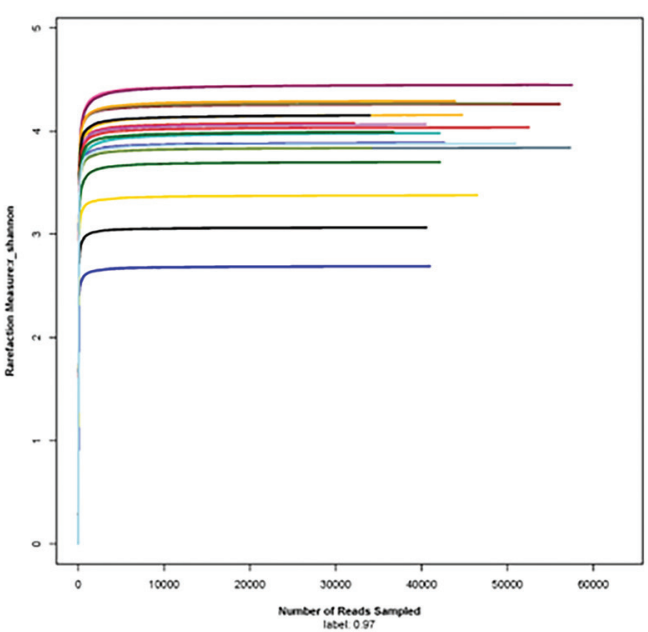

C

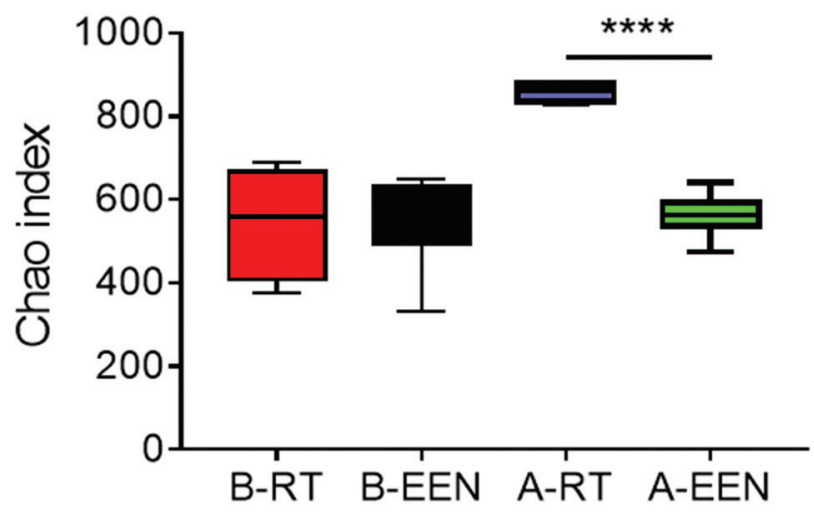

E
B

\section{Rank-abundance distribution curve}

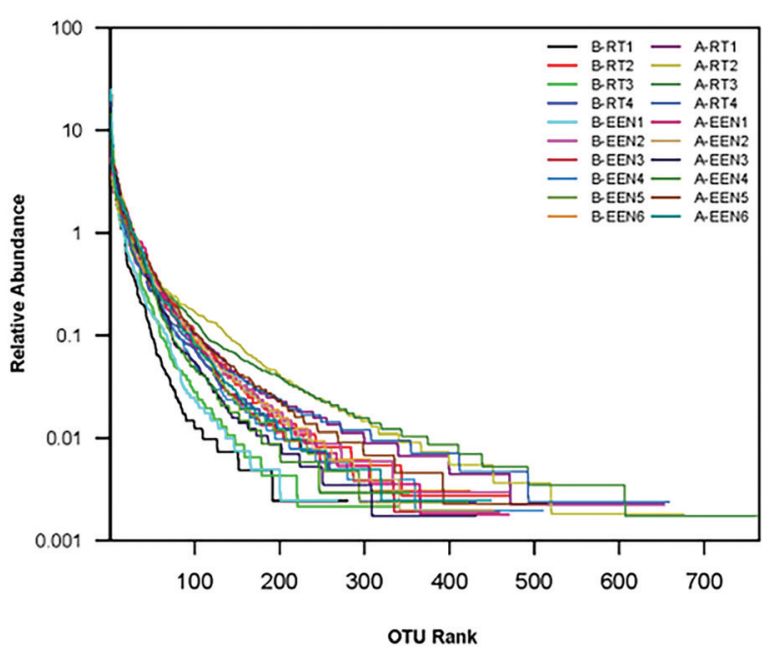

$\mathrm{D}$

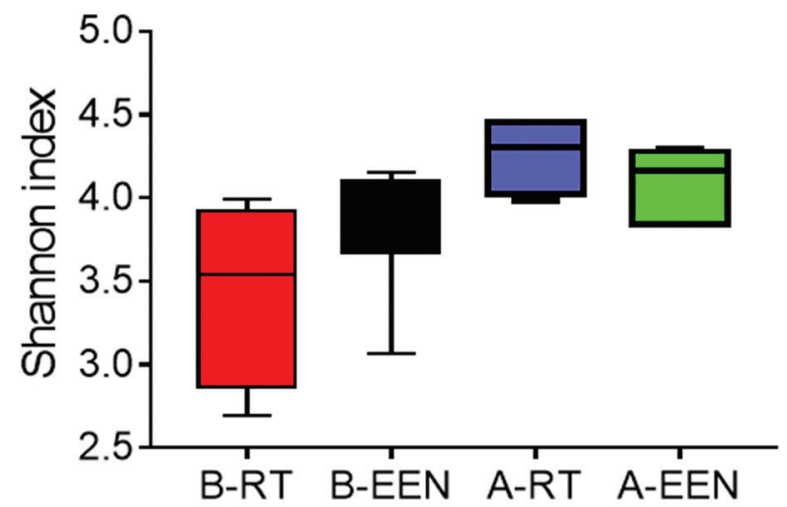

F

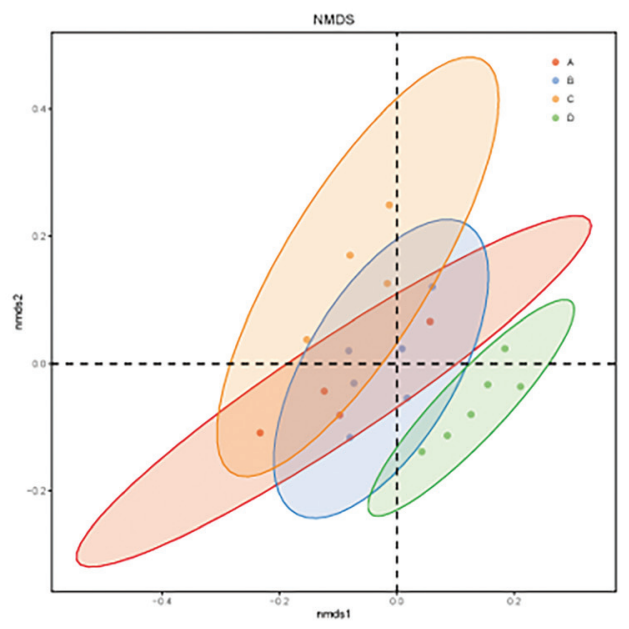

Fig. 3 EEN feeding modulates the diversity of gut microbiota. Fecal pellets were collected from both groups of mice before and after RT or EEN feeding. Microbial DNA was extracted and amplified for $16 \mathrm{~S}$ rRNA sequencing. The Shannon-Wiener curve (A) and rank-abundance distribution curve (B) among all samples. $\alpha$-Diversity was measured by the Chao index (C) and Shannon index (D). (E and F) Plots of UniFrac distance-based principal coordination analysis (PCOA) and nonmetric multidimensional scaling (NMDS), respectively. Each point represents an individual sample and the same color indicates a specific group. $(P=0.001) \mathrm{B}-\mathrm{RT}$, before RT; B-EEN, before EEN; A-RT, after RT; A-EEN, after EEN. A, B, C and D represent the groups of B-RT, B-EEN, A-RT and A-EEN, respectively. ${ }^{* * * * P}<0.0001$. 
A
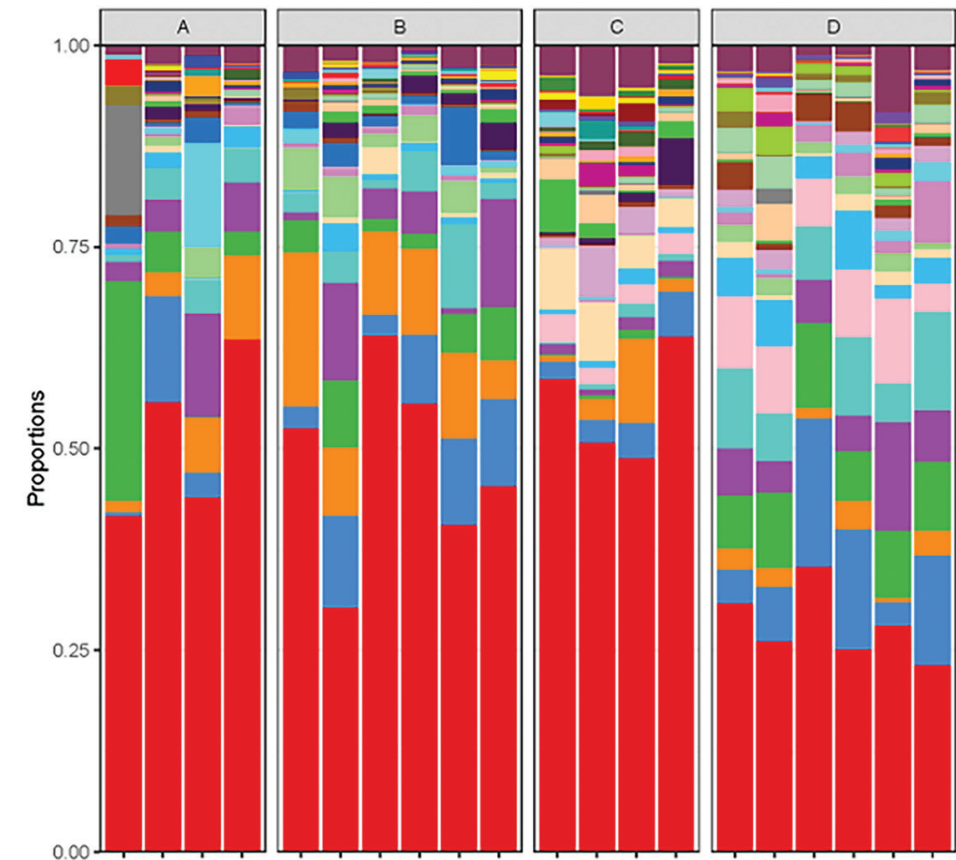

Eacteradales S24-7 group_norank

Alloprevdellia

Lactobacillus

Parabacteroides

Bacterades

Aistipes

Allobaculum

Odoribacter

Lachnospiraceae NK4A136 group

Faecalibaculum

Rikenellaceae RC9 gut group

[Eubacterium] fissicatena group

Lachnospiraceae_uncultured

Escherichia-Shigella

Parasutterella

Prevoellaceae UCG-001

Ruminococcace ae UCG-014

Ruminiclostridium 9

Lachnoclostridium

Rikenella

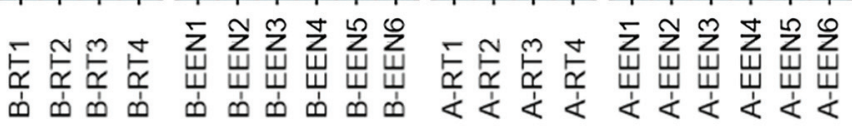

B
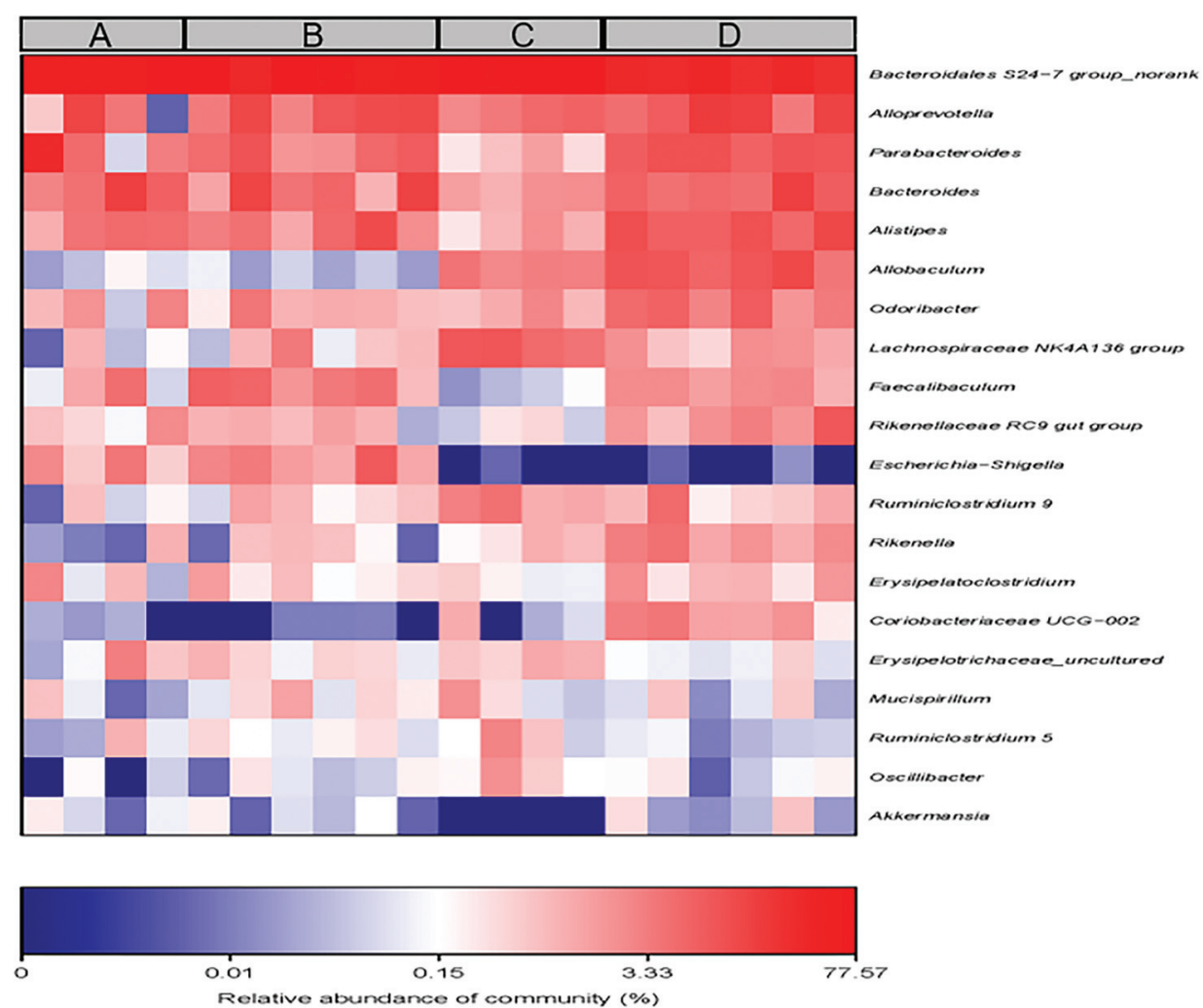

Fig. 4 EEN feeding alters the compositions of the dominant fecal microbiota. (A) Microbial community bar plot at the genus level. (B) Heatmap of the gut microbiota in different groups at the genus level. A, B, C and D represent the groups of B-RT, B-EEN, A-RT and A-EEN, respectively. 
A

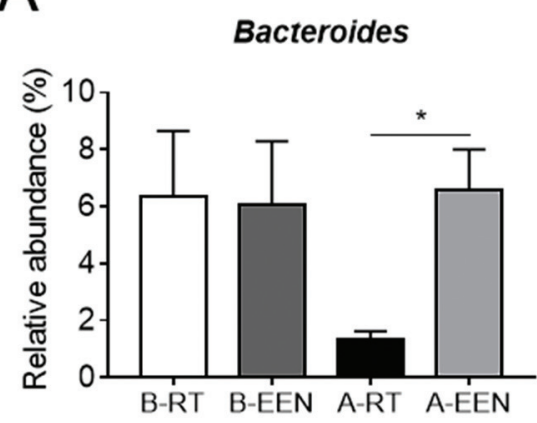

D

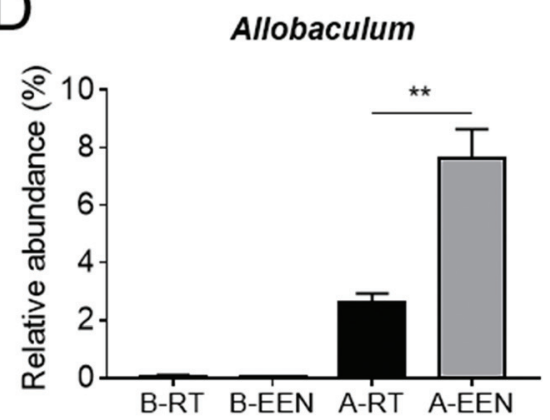

$G$

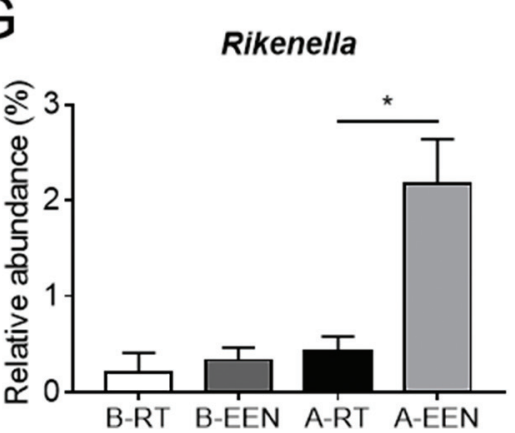

B

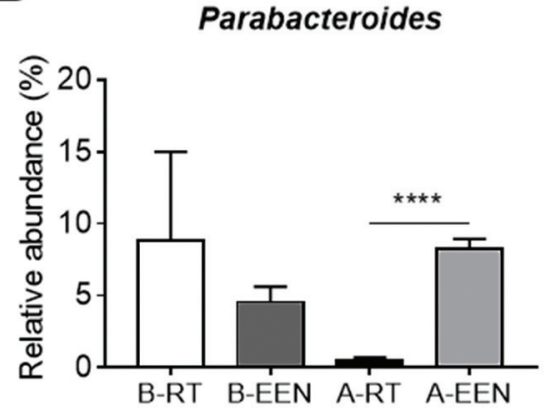

E

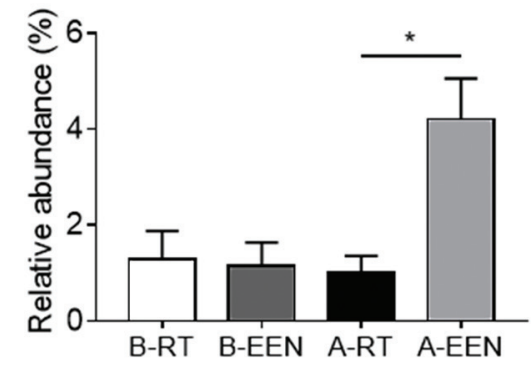

$\mathrm{H}$

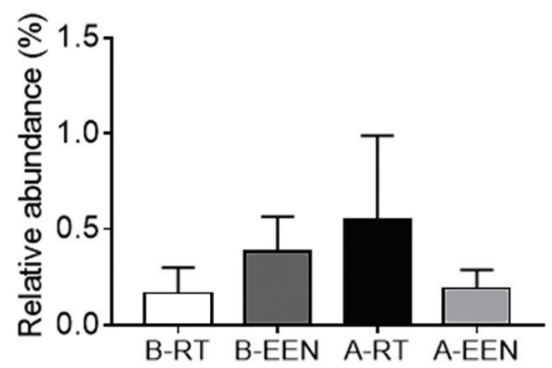

C

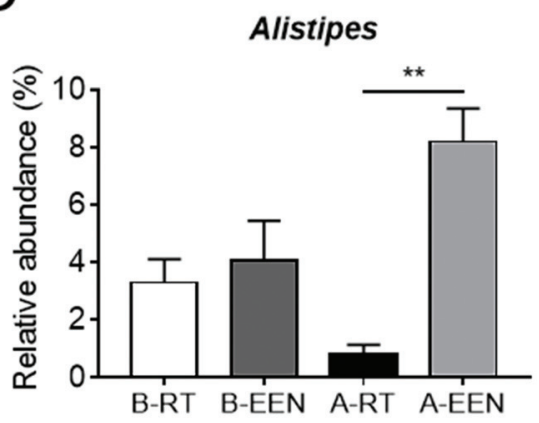

F

Coriobacteriaceae UCG-002

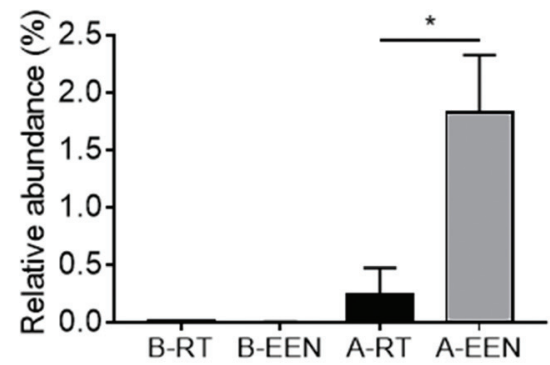

Escherichia-Shigella

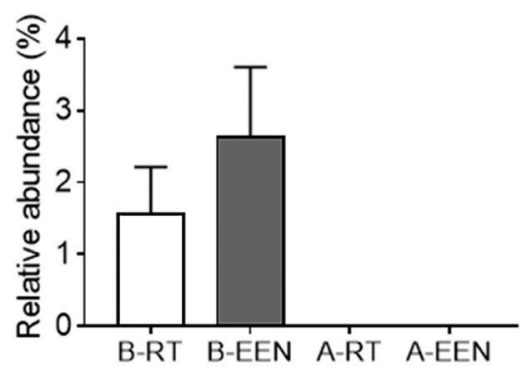

Fig. 5 Comparison of the relative abundance at the genus levels among different groups. Relative abundance of Bacteroides (A), Parabacteroides (B), Alistipes (C), Allobaculum (D), Odoribacter (E), Coriobacteriaceae UCG-002 (F), Rikenella (G), Mucispirillum (H) and Escherichia-Shigella (I) among different groups. B-RT, before RT; B-EEN, before EEN; A-RT, after RT; A-EEN, after EEN. ${ }^{*} P<0.05,{ }^{* \star} P<0.01$ and ${ }^{\star * * \star} P<0.0001$.

present study indicate that the distinct microbial signatures are tightly associated with EEN manipulation.

EEN alleviates intestinal mucosal inflammation of TNBSinduced murine colitis through remodeling the gut microbiota

Given that the long-term EEN could change the gut microbial composition and promote the growth of some beneficial bacteria, we investigated whether altered gut ecology by EEN could confer potential protective effects against intestinal inflammation. We then employed an acute TNBS-induced colitis model to examine whether long-term EEN could enhance tolerance and resistance to TNBS-induced colitis in mice (Fig. 7A). As shown in Fig. 7B-E, EEN feeding mice showed milder colitis characterized by a slight decrease of weight change, lower levels of the disease activity index and alleviated histological scores after the administration of TNBS in comparison with the RT feeding mice. Representative H\&E images of the colon sections revealed less structural damage and inflammatory cell infiltrates in the EEN feeding mice than those in the RT feeding controls (Fig. 7E and F). The levels of inflammatory cytokines in the colonic tissues which reflect mucosal inflammatory responses such as TNF- $\alpha$ and IL- 6 were lower in the EEN feeding TNBS-induced colitis mice than in the RT feeding controls (Fig. 7G and H). Furthermore, we collected the fecal samples for the detection of the levels of IgAand IgG-coated bacteria and free immunoglobulins. As expected, IgA- and IgG-coated bacteria and the concentrations of soluble IgA and IgG increased in the feces of TNBS-induced colitis mice but remarkably decreased after EEN feeding (Fig. 7I and J). Besides, qRT-PCR analysis of fecal 16S rRNA 
A

B

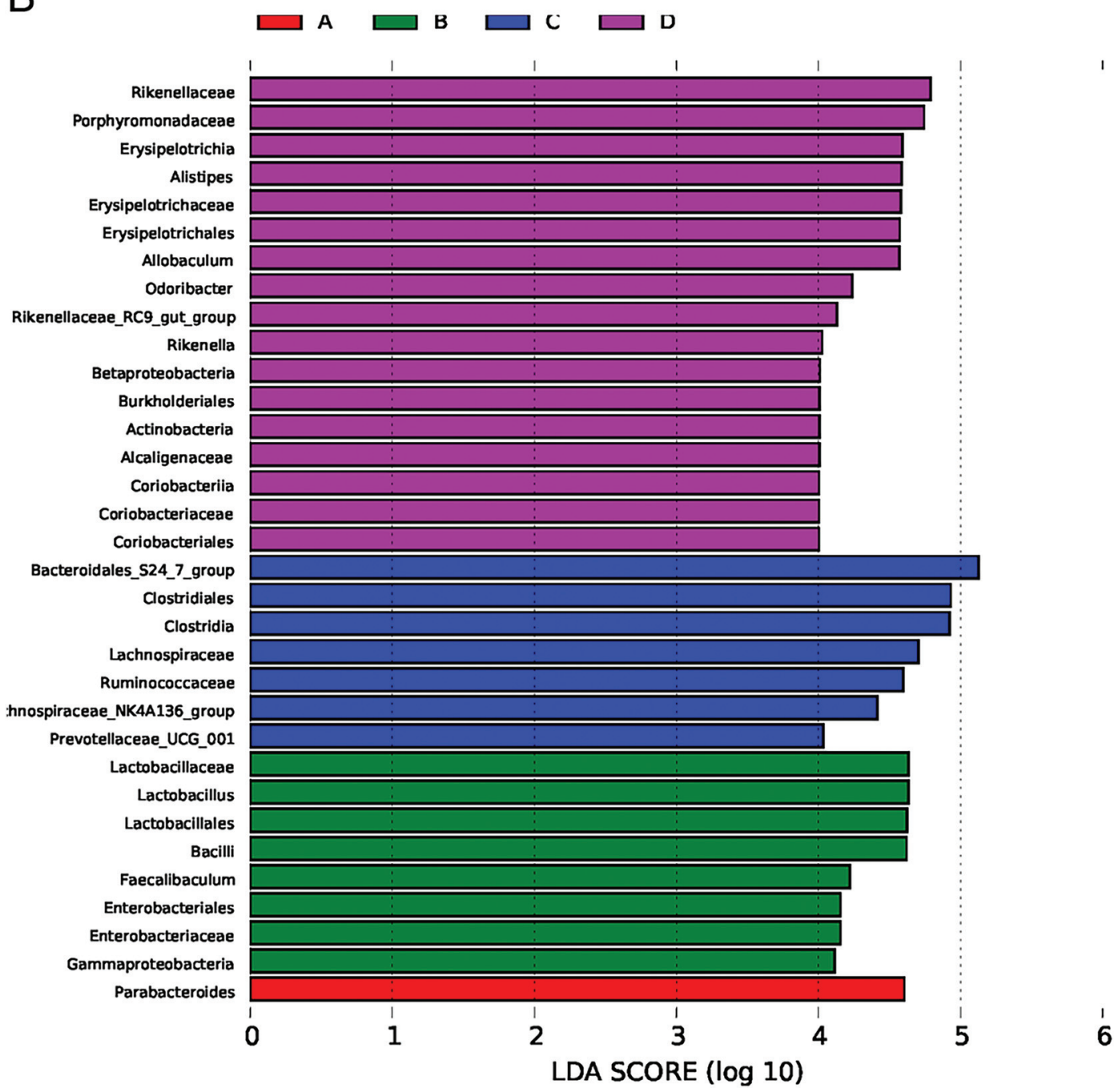

Fig. 6 LEfSe analysis of the abundant taxa among four groups. (A) LEfSe cladogram plot of the discriminative microbial taxa. The color represents the mouse group and the size of the circle represents the relative abundance of the taxa. (B) LDA scores of the taxa with significant effects. Differences were shown by the color of the most abundant class. The diameter of each circle is proportional to the taxon's abundance. A, B, C and D represent the groups of B-RT, B-EEN, A-RT and A-EEN, respectively. 
A

\begin{tabular}{|c|c|c|c|}
\hline Regular Diet & Breastfeeding & Standard mouse chow and water & TNBS \\
\hline Exclusive Enteral Nutritior & Breastfeeding & Exclusive Enteral Nutrition & TNBS \\
\hline & & & \\
\hline & & & \\
\hline
\end{tabular}

B

\section{C}
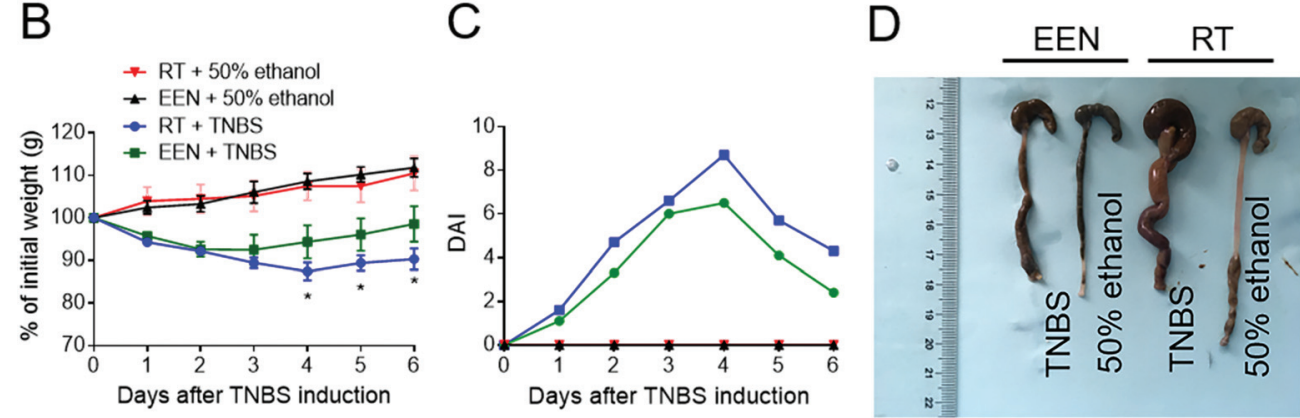

E

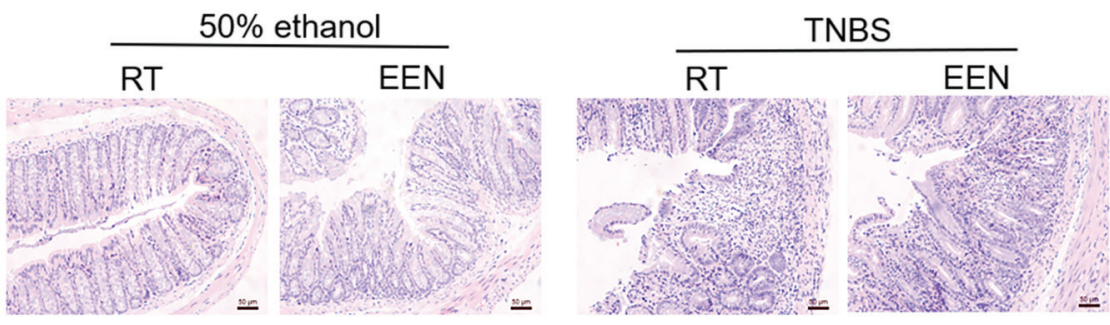

F

G
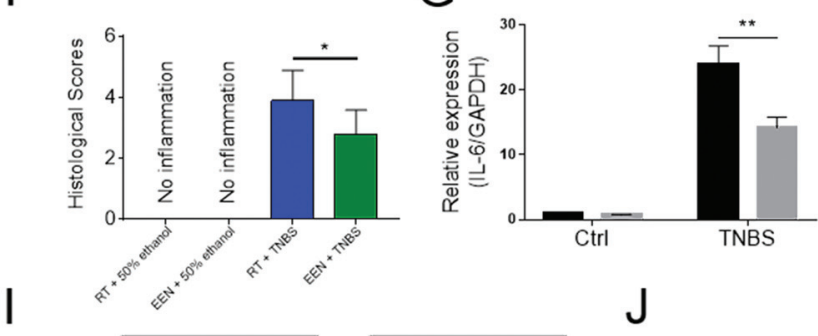

$\mathrm{H}$
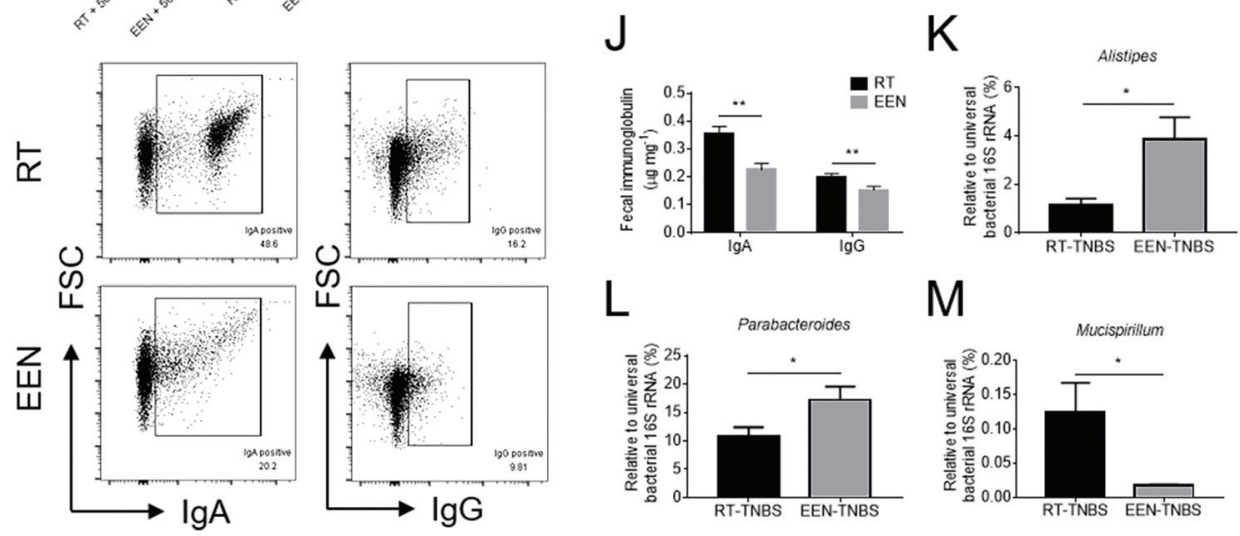

Fig. 7 Long-term EEN feeding prevents TNBS-induced colitis in mice through remodeling the gut microbiota. Mice growing up through feeding with RT or EEN at the age of 8 weeks as described in Fig. $1 \mathrm{~A}$ were used to establish an experimental colitis model induced by a rectal administration with TNBS ( $n=10$ in each group). (A) Schematic diagram of the experimental design. (B) Changes of body weight during a period of observation were shown as percent of the initial body weight at the beginning of the experiments. ${ }^{*} P<0.05$ versus the RT-treated group on the same day. (C) Disease activity index scores were recorded daily until day 6 after TNBS administration. (D) Gross morphology of the large bowels in different groups on day 6. (E) Representative H\&E images of the distal colon sections. Scale bars, $50 \mu \mathrm{m}$. (F) Histological scores were calculated from the colon sections as indicated. (G and H) The levels of IL- 6 and TNF- $\alpha$ mRNA in the colon tissues were detected by qRT-PCR. (I and J) Fecal pellets from RT or EEN feeding colitic mice were collected for the measurement of the FACS profiles of IgA- and IgG-coated bacteria as well as soluble IgA and IgG. $(\mathrm{K}-\mathrm{M})$ The relative abundance of selected bacteria in the feces sample from the RT or EEN treatment group is shown in the histogram. ${ }^{*}<0.05$ and $* * P<0.01$. 
demonstrated that the beneficial bacteria such as Parabacteroides and Alistipes were increased, while bacteria prone to colitis like Mucispirillum were decreased in the EEN feeding mice (Fig. 7K-M). Taken together, these results suggest that the EEN feeding mice are more tolerant to TNBSinduced colitis and that the EEN-induced beneficial gut ecology may account for the protective effects on the development of colitis.

\section{Discussion}

In the present study, we demonstrated that feeding with EEN modulated the composition of gut microbiota and the altered gut ecology may confer a protective role in TNBS-induced colitis. For the first time, we confirmed that mice treated with long-term EEN had normal growth and immune cell development, suggesting the possibilities of long-term EEN prescription. The restructuring of the gut microbiota composition including an increase of beneficial bacteria and a decrease of detrimental bacteria may strengthen the tolerance and resistance towards TNBS-induced colitis.

Inflammatory bowel diseases, including $\mathrm{CD}$ and ulcerative colitis (UC), are multi-factor mediated intestinal inflammatory diseases. Impaired intestinal homeostasis is a prominent characteristic of CD. Gut ecology consists of host intestinal tissues and gut microbiota and its derived metabolites, all contributing to a balanced gut environment. Recent advances in the field of microbiome and IBD have demonstrated that microbiome plays an important role in the pathogenesis of IBD. Numerous studies have indicated the differences in the composition and functionality of gut microbiota between patients with IBD and healthy individuals. ${ }^{36}$ Previous studies have demonstrated that the abundances of Bacteroides and Firmicutes such as Faecalibacterium prausnitzii are decreased in the feces from IBD patients, ${ }^{37-39}$ whereas members of the Proteobacteria phylum, such as Escherichia coli strains, are increased. Despite this gain in knowledge from the existing studies, we are still not clear whether there are one or several specific pathobionts which can cause IBD under specific conditions. Large gaps in our understanding of mechanistic associations between microbiota and IBD still remain. Therefore, discovery and verification of a specific individual pathogenic bacterial species related to the disease will certainly make a difference. In the past decades, microbial strategies including probiotics, prebiotics, and fecal microbiota transplantation (FMT) aiming to correct gut dysbiosis and encourage a diverse ecosystem have been attempted and achieved some benefits. Diet or meal, as an alternative environmental factor, influences the composition and functionality of the intestinal microbiome, ${ }^{40}$ barrier function and mucosal immune response. ${ }^{7}$ Dietary manipulations, as the most desirable therapeutic strategies, truly represent a promising direction in the management of IBD.

EEN is a dietary treatment with a formula-based diet involving the use of a complete liquid diet to provide $100 \%$ of caloric needs and exclusion of food for 6-8 weeks. The first published report with respect to the use of EEN in pediatric CD dated from the 1980s. ${ }^{41}$ Thereafter, there have been more and more research studies on EEN. In the late 1990s, EEN was eventually confirmed effective, and has been recommended as a first-line therapy in treatment guidelines to treat pediatric CD since the 2000s. EEN is as effective as corticosteroids in the treatment of pediatric $\mathrm{CD}$, with approximately up to $80 \%$ of pediatric patients achieving remission. Obvious benefits such as avoidance of corticosteroid associated side effects, improvement of nutritional status and bone health and reducing the need for surgery make it occupy an important position in the treatment of pediatric CD. ${ }^{42}$ Experimental evidence has shown that EEN plays a part in the regulation of intestinal inflammatory responses and barrier function. It is unambiguous how IBD is associated with compositional and metabolic changes of the intestinal microbiota. The intricate relationships between the gut microbiota and host make it difficult to precisely identify the gut microbiota as a cause or effect with IBD. ${ }^{43}$ Despite the two major roles of EEN, the most central mechanism of action of EEN may be attributed to the reduction in the gut microbial diversity. ${ }^{9,10,12}$ However, the exact mechanisms of how EEN exerts effects remain incompletely understood and the relationships between diet-induced remission, microbiome and colitis are still unclear. For example, the questions if long-term EEN could regulate the growth, development and immune dynamics of an individual as well as physiological composition of gut microbiota so as to improve individual tolerance and resistance to inflammatory stimuli remain to be answered.

In order to illustrate the long-term effects of EEN, we firstly fed mice with sole EEN suspensions from weaning to adulthood. We found that EEN feeding mice have normal growth and immune cell development, suggesting the possibility of long-term use. Given that aberrant immune responses accompanied by tremendously released inflammatory cytokines in the intestine are the caustive factor of IBD pathogenesis, ${ }^{44}$ we analyzed the mRNA levels of some relevant inflammatory cytokines in the intestinal mucosa and found no difference after the EEN treatment, though showing a declining trend (data not shown). These results suggested that mice fed with EEN had a normal intestinal immune microenvironment. Numerous studies have confirmed that host diet can shape the immune recognition of the microbiota. For example, dietary undernutrition was demonstrated to induce a metabolic change, resulting in differential IgA-bacterial interactions. ${ }^{45}$ We then detected and found decreased levels of free immunoglobulins and immunoglobulin-coated bacteria in the feces. Previous studies have suggested that IgA coating identifies colitogenic bacteria that preferentially drive intestinal inflammation. ${ }^{28}$ Our group also found that IgAcoated bacteria in the feces and sera are closely associated with the disease activity of IBD patients. ${ }^{29}$ Therefore, we proposed that the decreased levels of immunoglobulins as well as the coated bacteria may be a result of decreased detrimental bacteria. 
The preliminary finding that EEN feeding changed the levels of free immunoglobulins and immunoglobulin-coated bacteria forced us to probe whether long-term EEN could modulate the composition of the gut microbiota. 16S rRNA sequencing was utilized to reveal the alteration in the gut microbial composition. Our animal study found that fecal microbiota from the EEN feeding group showed decreased levels of $\alpha$-diversity compared with that in the RT feeding group. This result is consistent with previous research studies which demonstrated that treatment with EEN further decreased microbial diversity in spite of improved disease activity in pediatric CD. ${ }^{46}$ We further compared the compositional differences in the gut microbiota between the RT and EEN feeding groups. PCoA plots indicated evident separation of the gut microbiota composition from the two groups after feeding. Moreover, the microbial community bar plot and heatmap showed the community structural differences in detail. The opportunistic pathogens such as Mucispirillum and Escherichia-Shigella markedly decreased, while the levels of beneficial bacteria including Bacteroides, Parabacteroides, Alistipes, Allobaculum, Odoribacter, Coriobacteriaceae UCG-002 and Rikenella increased. These structural changes of the gut microbiota exerted by EEN treatment resemble previous findings acquired from human studies. ${ }^{47}$ Based on the above findings, we investigated whether mice, after feeding with longterm EEN, would possess enhanced resistance and tolerance upon TNBS insults and whether changes in the gut microbiota would make a difference. As expected, we observed that the EEN feeding group exhibited less severe colitis and decreased levels of fecal soluble immunoglobulin as well as an increase of some beneficial bacteria relative to the RT feeding group. Although the definitive causality of the gut microbiota and colitis remains undetermined, plenty of evidence indicates that microbiota can be transferred and initiate disease onset in the susceptible host. ${ }^{48} \mathrm{~A}$ monotonous dietary intake has been demonstrated to provide protection against acute DSS colitis, suggesting that increased dietary diversity may contribute to the emergence of IBD. ${ }^{49}$ Based on the present results, we anticipated that the increased tolerance may be attributed to the reduced nutrient load and boosted production of short chain fatty acids by the gut microbiota with altered functional activity, in agreement with a previous human study. ${ }^{50}$ A less sensitive but more balanced gut ecosystem with strong regulatory ability contributes to an alleviated phenotype in the TNBSinduced colitis model. Nonetheless, a more mechanistic dissection of the impact of EEN on the structure and functional activity of the gut microbiota is still needed. Collectively, these data suggest that the alleviated phenotype by EEN may be attributed at least in part to the improved dysbiosis of the gut microbiota.

\section{Conclusions}

In conclusion, the above results clarify the impact of long-term EEN feeding on the community composition of the gut micro- biota, and this significant change in the gut microbiota may confer a protective role in TNBS-induced colitis. Our data suggest that the beneficial effects of EEN on TNBS colitis are mediated, at least in part, through remodeling the gut microbiota. There are still a lot of questions pertaining to the gut microbiota and its relationship with health and disease, which warrants further research studies. Manipulation of the gut microbiome in gut ecology through different methods represents a novel and powerful approach in the management of health and disease, such as IBD.

\section{Abbreviations}

$\begin{array}{ll}\text { CD } & \text { Crohn's disease } \\ \text { CDED } & \text { Crohn's disease exclusion diet } \\ \text { EEN } & \text { Exclusive enteral nutrition } \\ \text { FODMAP } & \text { Fermentable oligosaccharides, disaccharides, } \\ & \text { monosaccharides and polyols } \\ \text { IBD } & \text { Inflammatory bowel disease } \\ \text { OTUs } & \text { Operational taxonomic units } \\ \text { PEN } & \text { Partial enteral nutrition } \\ \text { RT } & \text { Regular diet } \\ \text { SCD } & \text { Specific carbohydrate diet } \\ \text { TNBS } & \text { Trinitrobenzenesulfonic acid }\end{array}$

\section{Ethical statement}

All animal procedures were performed in accordance with the Guidelines for Care and Use of Laboratory Animals of Tongji University and approved by the Animal Ethics Committee of Shanghai Tenth People's Hospital (SHDSYY-2020-3914; Shanghai, China).

\section{Author contributions}

Conceptualization, L. F. and W. W.; investigation, G. L., X. W., X. G. and R. L.; writing - original draft, G. L., L. C., M. S. and J. J.; and writing - review and editing, G. L., Z. L., L. F. and W. W. All authors have read and agreed to the published version of the manuscript.

\section{Conflicts of interest}

The authors have no conflicts of interest to disclose.

\section{Acknowledgements}

This work was supported by grants from the National Natural Science Foundation of China (82070562, 9194230064, 91942312 and 81800486) and the Shanghai Rising-Star Program (20QA1407700). 


\section{References}

1 R. J. Xavier and D. K. Podolsky, Unravelling the pathogenesis of inflammatory bowel disease, Nature, 2007, 448, 427434.

2 M. Joossens, G. Huys, M. Cnockaert, V. De Preter, K. Verbeke, P. Rutgeerts, P. Vandamme and S. Vermeire, Dysbiosis of the faecal microbiota in patients with Crohn's disease and their unaffected relatives, Gut, 2011, 60, 631637.

3 D. Gevers, S. Kugathasan, L. A. Denson, Y. Vázquez-Baeza, W. Van Treuren, B. Ren, E. Schwager, D. Knights, S. J. Song, M. Yassour, X. C. Morgan, A. D. Kostic, C. Luo, A. González, D. McDonald, Y. Haberman, T. Walters, S. Baker, J. Rosh, M. Stephens, M. Heyman, J. Markowitz, R. Baldassano, A. Griffiths, F. Sylvester, D. Mack, S. Kim, W. Crandall, J. Hyams, C. Huttenhower, R. Knight and R. J. Xavier, The treatment-naive microbiome in new-onset Crohn's disease, Cell Host Microbe, 2014, 15, 382-392.

4 B. D. Muegge, J. Kuczynski, D. Knights, J. C. Clemente, A. González, L. Fontana, B. Henrissat, R. Knight and J. I. Gordon, Diet drives convergence in gut microbiome functions across mammalian phylogeny and within humans, Science, 2011, 332, 970-974.

5 S. Mehandru and J. F. Colombel, The intestinal barrier, an arbitrator turned provocateur in IBD, Nat. Rev. Gastroenterol. Hepatol., 2021, 18, 83-84.

6 P. F. van Rheenen, M. Aloi, A. Assa, J. Bronsky, J. C. Escher, U. L. Fagerberg, M. Gasparetto, K. Gerasimidis, A. Griffiths, P. Henderson, S. Koletzko, K. L. Kolho, A. Levine, J. van Limbergen, F. J. M. de Carpi, V. M. Navas-López, S. Oliva, L. de Ridder, R. K. Russell, D. Shouval, A. Spinelli, D. Turner, D. Wilson, E. Wine and F. M. Ruemmele, The Medical Management of Paediatric Crohn's Disease: an ECCO-ESPGHAN Guideline Update, J. Crohn's Colitis, 2021, 15(2), 171-194.

7 J. J. Ashton, J. Gavin and R. M. Beattie, Exclusive enteral nutrition in Crohn's disease: Evidence and practicalities, Clin. Nutr., 2019, 38, 80-89.

8 S. Gatti, T. Galeazzi, E. Franceschini, R. Annibali, V. Albano, A. K. Verma, M. De Angelis, M. E. Lionetti and C. Catassi, Effects of the Exclusive Enteral Nutrition on the Microbiota Profile of Patients with Crohn's Disease: A Systematic Review, Nutrients, 2017, 9, 832.

9 C. Quince, U. Z. Ijaz, N. Loman, A. M. Eren, D. Saulnier, J. Russell, S. J. Haig, S. T. Calus, J. Quick, A. Barclay, M. Bertz, M. Blaut, R. Hansen, P. McGrogan, R. K. Russell, C. A. Edwards and K. Gerasimidis, Extensive Modulation of the Fecal Metagenome in Children With Crohn's Disease During Exclusive Enteral Nutrition, Am. J. Gastroenterol., 2015, 110, 1718-1729. quiz 1730.

10 S. T. Leach, H. M. Mitchell, W. R. Eng, L. Zhang and A. S. Day, Sustained modulation of intestinal bacteria by exclusive enteral nutrition used to treat children with Crohn's disease, Aliment. Pharmacol. Ther., 2008, 28, 724-733.
11 X. C. Morgan, T. L. Tickle, H. Sokol, D. Gevers, K. L. Devaney, D. V. Ward, J. A. Reyes, S. A. Shah, N. LeLeiko, S. B. Snapper, A. Bousvaros, J. Korzenik, B. E. Sands, R. J. Xavier and C. Huttenhower, Dysfunction of the intestinal microbiome in inflammatory bowel disease and treatment, Genome Biol., 2012, 13, R79.

12 N. O. Kaakoush, A. S. Day, S. T. Leach, D. A. Lemberg, S. Nielsen and H. M. Mitchell, Effect of exclusive enteral nutrition on the microbiota of children with newly diagnosed Crohn's disease, Clin. Transl. Gastroenterol., 2015, 6, e71.

13 N. O. Kaakoush, A. S. Day, S. T. Leach, D. A. Lemberg and H. M. Mitchell, Reduction in Gut Microbial Diversity as a Mechanism of Action of Exclusive Enteral Nutrition, Am. J. Gastroenterol., 2016, 111, 1033.

14 A. Wedrychowicz, K. Kowalska-Duplaga, U. JedynakWasowicz, S. Pieczarkowski, M. Sladek, P. Tomasik and K. Fyderek, Serum concentrations of VEGF and TGF- $\beta 1$ during exclusive enteral nutrition in IBD, J. Pediatr. Gastroenterol. Nutr., 2011, 53, 150-155.

15 J. M. Fell, M. Paintin, F. Arnaud-Battandier, R. M. Beattie, A. Hollis, P. Kitching, A. Donnet-Hughes, T. T. MacDonald and J. A. Walker-Smith, Mucosal healing and a fall in mucosal pro-inflammatory cytokine mRNA induced by a specific oral polymeric diet in paediatric Crohn's disease, Aliment. Pharmacol. Ther., 2000, 14, 281-289.

16 T. Yamamoto, M. Nakahigashi, S. Umegae, T. Kitagawa and K. Matsumoto, Impact of elemental diet on mucosal inflammation in patients with active Crohn's disease: cytokine production and endoscopic and histological findings, Inflammatory Bowel Dis., 2005, 11, 580-588.

17 N. S. de Jong, S. T. Leach and A. S. Day, Polymeric formula has direct anti-inflammatory effects on enterocytes in an in vitro model of intestinal inflammation, Dig. Dis. Sci., 2007, 52, 2029-2036.

18 I. R. Sanderson, P. Boulton, I. Menzies and J. A. WalkerSmith, Improvement of abnormal lactulose/rhamnose permeability in active Crohn's disease of the small bowel by an elemental diet, Gut, 1987, 28, 1073-1076.

19 L. Nahidi, A. S. Day, D. A. Lemberg and S. T. Leach, Differential effects of nutritional and non-nutritional therapies on intestinal barrier function in an in vitro model, J. Gastroenterol., 2012, 47, 107-117.

20 L. Nahidi, S. T. Leach, H. M. Mitchell, N. O. Kaakoush, D. A. Lemberg, J. S. Munday, K. Huinao and A. S. Day, Inflammatory bowel disease therapies and gut function in a colitis mouse model, BioMed Res. Int., 2013, 2013, 909613.

21 M. H. Alhagamhmad, A. S. Day, D. A. Lemberg and S. T. Leach, Exploring and Enhancing the AntiInflammatory Properties of Polymeric Formula, JPEN, J. Parenter. Enteral Nutr., 2017, 41, 436-445.

22 J. L. Grogan, D. H. Casson, A. Terry, G. C. Burdge, W. ElMatary and A. M. Dalzell, Enteral feeding therapy for newly diagnosed pediatric Crohn's disease: a double-blind randomized controlled trial with two years follow-up, Inflammatory Bowel Dis., 2012, 18, 246-253. 
23 T. Johnson, S. Macdonald, S. M. Hill, A. Thomas and M. S. Murphy, Treatment of active Crohn's disease in children using partial enteral nutrition with liquid formula: a randomised controlled trial, Gut, 2006, 55, 356-361.

24 D. Lee, R. N. Baldassano, A. R. Otley, L. Albenberg, A. M. Griffiths, C. Compher, E. Z. Chen, H. Li, E. Gilroy, L. Nessel, A. Grant, C. Chehoud, F. D. Bushman, G. D. Wu and J. D. Lewis, Comparative Effectiveness of Nutritional and Biological Therapy in North American Children with Active Crohn's Disease, Inflammatory Bowel Dis., 2015, 21, 1786-1793.

25 Y. Ge, M. Sun, W. Wu, C. Ma, C. Zhang, C. He, J. Li, Y. Cong, D. Zhang and Z. Liu, MicroRNA-125a suppresses intestinal mucosal inflammation through targeting ETS-1 in patients with inflammatory bowel diseases, J. Autoimmun., 2019, 101, 109-120.

26 W. Wu, M. Sun, F. Chen, A. T. Cao, H. Liu, Y. Zhao, X. Huang, Y. Xiao, S. Yao, Q. Zhao, Z. Liu and Y. Cong, Microbiota metabolite short-chain fatty acid acetate promotes intestinal IgA response to microbiota which is mediated by GPR43, Mucosal Immunol., 2017, 10, 946956.

27 J. G. Caporaso, J. Kuczynski, J. Stombaugh, K. Bittinger, F. D. Bushman, E. K. Costello, N. Fierer, A. G. Peña, J. K. Goodrich, J. I. Gordon, G. A. Huttley, S. T. Kelley, D. Knights, J. E. Koenig, R. E. Ley, C. A. Lozupone, D. McDonald, B. D. Muegge, M. Pirrung, J. Reeder, J. R. Sevinsky, P. J. Turnbaugh, W. A. Walters, J. Widmann, T. Yatsunenko, J. Zaneveld and R. Knight, QIIME allows analysis of high-throughput community sequencing data, Nat. Methods, 2010, 7, 335-336.

28 N. W. Palm, M. R. de Zoete, T. W. Cullen, N. A. Barry, J. Stefanowski, L. Hao, P. H. Degnan, J. Hu, I. Peter, W. Zhang, E. Ruggiero, J. H. Cho, A. L. Goodman and R. A. Flavell, Immunoglobulin A coating identifies colitogenic bacteria in inflammatory bowel disease, Cell, 2014, 158, 1000-1010.

29 R. Lin, H. Chen, W. Shu, M. Sun, L. Fang, Y. Shi, Z. Pang, W. Wu and Z. Liu, Clinical significance of soluble immunoglobulins $A$ and $G$ and their coated bacteria in feces of patients with inflammatory bowel disease, J. Transl. Med., 2018, 16, 359.

30 R. Dziarski, S. Y. Park, D. R. Kashyap, S. E. Dowd and D. Gupta, Pglyrp-Regulated Gut Microflora Prevotella falsenii, Parabacteroides distasonis and Bacteroides eggerthii Enhance and Alistipes finegoldii Attenuates Colitis in Mice, PLoS One, 2016, 11, e0146162.

31 B. J. Parker, P. A. Wearsch, A. C. M. Veloo and A. Rodriguez-Palacios, The Genus Alistipes: Gut Bacteria With Emerging Implications to Inflammation, Cancer, and Mental Health, Front. Immunol., 2020, 11, 906.

32 M. Kverka, Z. Zakostelska, K. Klimesova, D. Sokol, T. Hudcovic, T. Hrncir, P. Rossmann, J. Mrazek, J. Kopecny, E. F. Verdu and H. Tlaskalova-Hogenova, Oral administration of Parabacteroides distasonis antigens attenuates experimental murine colitis through modulation of immu- nity and microbiota composition, Clin. Exp. Immunol., 2011, 163, 250-259.

33 B. Lamas, M. L. Richard, V. Leducq, H. P. Pham, M. L. Michel, G. Da Costa, C. Bridonneau, S. Jegou, T. W. Hoffmann, J. M. Natividad, L. Brot, S. Taleb, A. Couturier-Maillard, I. Nion-Larmurier, F. Merabtene, P. Seksik, A. Bourrier, J. Cosnes, B. Ryffel, L. Beaugerie, J. M. Launay, P. Langella, R. J. Xavier and H. Sokol, CARD9 impacts colitis by altering gut microbiota metabolism of tryptophan into aryl hydrocarbon receptor ligands, Nat. Med., 2016, 22, 598-605.

34 M. Göker, S. Gronow, A. Zeytun, M. Nolan, S. Lucas, A. Lapidus, N. Hammon, S. Deshpande, J. F. Cheng, S. Pitluck, K. Liolios, I. Pagani, N. Ivanova, K. Mavromatis, G. Ovchinikova, A. Pati, R. Tapia, C. Han, L. Goodwin, A. Chen, K. Palaniappan, M. Land, L. Hauser, C. D. Jeffries, E. M. Brambilla, M. Rohde, J. C. Detter, T. Woyke, J. Bristow, V. Markowitz, P. Hugenholtz, J. A. Eisen, N. C. Kyrpides and H. P. Klenk, Complete genome sequence of Odoribacter splanchnicus type strain (1651/6), Stand. Genomic Sci., 2011, 4, 200-209.

35 Y. Song, N. Malmuthuge, M. A. Steele and L. L. Guan, Shift of hindgut microbiota and microbial short chain fatty acids profiles in dairy calves from birth to pre-weaning, FEMS Microbiol. Ecol., 2018, 94(3).

36 C. Manichanh, N. Borruel, F. Casellas and F. Guarner, The gut microbiota in IBD, Nat. Rev. Gastroenterol. Hepatol., 2012, 9, 599-608.

37 H. Sokol, B. Pigneur, L. Watterlot, O. Lakhdari, L. G. Bermúdez-Humarán, J. J. Gratadoux, S. Blugeon, C. Bridonneau, J. P. Furet, G. Corthier, C. Grangette, N. Vasquez, P. Pochart, G. Trugnan, G. Thomas, H. M. Blottière, J. Doré, P. Marteau, P. Seksik and P. Langella, Faecalibacterium prausnitzii is an anti-inflammatory commensal bacterium identified by gut microbiota analysis of Crohn disease patients, Proc. Natl. Acad. Sci. U. S. A., 2008, 105, 16731-16736.

38 S. Miquel, R. Martín, O. Rossi, L. G. Bermúdez-Humarán, J. M. Chatel, H. Sokol, M. Thomas, J. M. Wells and P. Langella, Faecalibacterium prausnitzii and human intestinal health, Curr. Opin. Microbiol., 2013, 16, 255-261.

39 H. Sokol, P. Seksik, J. P. Furet, O. Firmesse, I. NionLarmurier, L. Beaugerie, J. Cosnes, G. Corthier, P. Marteau and J. Doré, Low counts of Faecalibacterium prausnitzii in colitis microbiota, Inflammatory Bowel Dis., 2009, 15, 11831189.

40 J. D. Lewis, E. Z. Chen, R. N. Baldassano, A. R. Otley, A. M. Griffiths, D. Lee, K. Bittinger, A. Bailey, E. S. Friedman, C. Hoffmann, L. Albenberg, R. Sinha, C. Compher, E. Gilroy, L. Nessel, A. Grant, C. Chehoud, H. Li, G. D. Wu and F. D. Bushman, Inflammation, Antibiotics, and Diet as Environmental Stressors of the Gut Microbiome in Pediatric Crohn's Disease, Cell Host Microbe, 2015, 18, 489-500.

41 C. L. Morin, M. Roulet, C. C. Roy and A. Weber, Continuous elemental enteral alimentation in children 
with Crohn's disease and growth failure, Gastroenterology, 1980, 79, 1205-1210.

42 F. M. Ruemmele, G. Veres, K. L. Kolho, A. Griffiths, A. Levine, J. C. Escher, J. Amil Dias, A. Barabino, C. P. Braegger, J. Bronsky, S. Buderus, J. Martín-de-Carpi, L. De Ridder, U. L. Fagerberg, J. P. Hugot, J. Kierkus, S. Kolacek, S. Koletzko, P. Lionetti, E. Miele, V. M. Navas López, A. Paerregaard, R. K. Russell, D. E. Serban, R. Shaoul, P. Van Rheenen, G. Veereman, B. Weiss, D. Wilson, A. Dignass, A. Eliakim, H. Winter and D. Turner, Consensus guidelines of ECCO/ESPGHAN on the medical management of pediatric Crohn's disease, J. Crohn's Colitis, 2014, 8, 1179-1207.

43 J. Ni, G. D. Wu, L. Albenberg and V. T. Tomov, Gut microbiota and IBD: causation or correlation?, Nat. Rev. Gastroenterol. Hepatol., 2017, 14, 573-584.

44 M. Friedrich, M. Pohin and F. Powrie, Cytokine Networks in the Pathophysiology of Inflammatory Bowel Disease, Immunity, 2019, 50, 992-1006.

45 K. E. Huus, K. C. Bauer, E. M. Brown, T. Bozorgmehr, S. E. Woodward, A. Serapio-Palacios, R. C. T. Boutin, C. Petersen and B. B. Finlay, Commensal Bacteria Modulate Immunoglobulin A Binding in Response to Host Nutrition, Cell Host Microbe, 2020, 27, 909-921.

46 K. Gerasimidis, M. Bertz, L. Hanske, J. Junick, O. Biskou, M. Aguilera, V. Garrick, R. K. Russell, M. Blaut,
P. McGrogan and C. A. Edwards, Decline in presumptively protective gut bacterial species and metabolites are paradoxically associated with disease improvement in pediatric Crohn's disease during enteral nutrition, Inflammatory Bowel Dis., 2014, 20, 861-871.

47 S. Gatti, T. Galeazzi, E. Franceschini, R. Annibali, V. Albano, A. K. Verma, M. De Angelis, M. E. Lionetti and C. Catassi, Effects of the Exclusive Enteral Nutrition on the Microbiota Profile of Patients with Crohn's Disease: A Systematic Review, Nutrients, 2017, 9, 832.

48 P. Cuív, J. Begun, S. Keely, P. J. Lewindon and M. Morrison, Towards an integrated understanding of the therapeutic utility of exclusive enteral nutrition in the treatment of Crohn's disease, Food Funct., 2016, 7, 17411751.

49 D. Nagy-Szakal, S. A. Mir, M. C. Ross, N. Tatevian, J. F. Petrosino and R. Kellermayer, Monotonous diets protect against acute colitis in mice: epidemiologic and therapeutic implications, J. Pediatr. Gastroenterol. Nutr., 2013, 56, 544-550.

50 B. Tjellström, L. Högberg, L. Stenhammar, K. E. Magnusson, T. Midtvedt, E. Norin and T. Sundqvist, Effect of exclusive enteral nutrition on gut microflora function in children with Crohn's disease, Scand. J. Gastroenterol., 2012, 47, 1454-1459. 